Review

\title{
Significance of intestinal microbiota in implementing metabolic effects of bariatric surgery
}

\author{
Marina A. Berkovskaya, Yulia P. Sych, Olesya Yu. Gurova, Valentin V. Fadeev \\ I.M. Sechenov First Moscow State Medical University (Sechenov University), Moscow, Russia
}

Received 11 February 2020, Revised 26 June 2020, Accepted 23 September 2020

(C) 2020, Berkovskaya M.A., Sych Y.P., Gurova O.Yu., Fadeev V.V.

(C) 2020, Russian Open Medical Journal

Abstract: Bariatric surgery is among successful methods of obesity treatment, with effects going beyond weight reduction alone, but rather involving improved glucose tolerance, along with control or remission of the type 2 diabetes mellitus. The precise mechanisms causing metabolic effects of bariatric surgery are not fully elucidated, even though substantial evidence suggest that they include changes in the gut microbiota, bile acid homeostasis, and the close interactions of these factors.

Intestinal microflora is directly involved in the energy metabolism of a host human. Obesity and type 2 diabetes mellitus are associated with certain changes in the species composition and diversity of intestinal microflora, which are considered important factors in the development and progression of these ailments. Bariatric surgery leads to significant and persistent changes in the composition of the intestinal microbiota, often bringing it closer to the characteristics of the microbiota of an average person with a normal weight. An important role in implementing the metabolic effects of bariatric surgery, primarily in the improvement of glucose metabolism, belongs to postoperative changes in homeostasis of bile acids. These changes imply close metabolism. Moreover, changes in the bile acid metabolism after bariatric surgery affect the microbiota of the host. Further study of these relationships would clarify the mechanisms underlying metabolic surgery, make it more predictable, targeted and controlled, as well as open new therapeutic targets in the treatment of obesity and associated conditions.

Keywords: obesity, gut microbiota, bariatric surgery, bile acids.

Cite as Berkovskaya MA, Sych YP, Gurova OYu, Fadeev VV. Significance of intestinal microbiota in implementing metabolic effects of bariatric surgery. Russian Open Medical Journal 2021; 10: e0112.

Correspondence to Marina A. Berkovskaya. Address: I.M. Sechenov First Moscow State Medical University (Sechenov University), 1 Pogodinskaya St., Moscow 119435, Russia. Phone: +79264530224. E-mail: abaitamar@gmail.com.

\section{Introduction}

Currently, obesity is the most widespread metabolic disorder in the world, virtually becoming an epidemy [1]. In 2014, about 2 billion people in the world were overweight and over 600 million were obese [2]. Excessive weight and obesity are important risk factors for personal and social health impairments, since they are associated with numerous comorbidities, such as type 2 diabetes mellitus (DM2), cardiovascular diseases and some types of malignant tumors [3]. Obesity is known as a major independent risk factor for the development of DM2, therefore its increasing incidence is undoubtedly attributed to expanding number of overweight and obese people. In 2015, there were 415 million of diabetics, and this number is expected to grow up to 642 million by 2040 [4]. According to the WHO data, over $90 \%$ patients with DM2 are obese or overweight [2]. In the modern world, DM has become a serious and real threat for public health with a significant economic burden for both individual patients and healthcare system of various countries.

The basic treatment for obesity involves life-style modifications, specifically a low-calorie diet, intensification of physical activity, and cognitive-behavioral therapy. Surgical methods have been widely used in case of ineffective conservative approaches, existing comorbidities or morbid stage of obesity.
The history of the bariatric surgery started nearly 60 years ago. Initially, its main goals were reduction and long-term maintenance of a body weight in the patients with morbid obesity. However, it has become quite obvious later, that the weigh-loss surgery was also causing a significant improvement of some metabolic parameters, including the carbohydrate metabolism [5, 6]. Many short-term and long-term studies demonstrated the efficacy of bariatric surgery in significant body weight reduction and diabetes control [7-9].

Currently, two most popular types of bariatric procedures for obesity and DM2 treatment are modified Roux-en-Y gastric bypass (RYGB) and sleeve gastrectomy (SG).

Initially, the metabolic consequences of bariatric procedures were explained solely by the mechanistic hypothesis based upon the restrictive and malabsorptive effects required for the weight loss occurrence. The former presumed reduction of the gastric volume, while the latter referred to a diminished area of absorption. However, in many studies, the remission of DM2 after gastric bypass surgery took place well before the substantial wight loss, implying an existence of other possible weigh-independent factors related to the metabolic effects of bariatric surgery. At present, intestinal hormones, bile acids, gut microflora, nervous system and some other potential factors are thought to play a key 
role in the development of metabolic benefits after bariatric manipulations [10]. Meanwhile, the exact mechanism of the beneficial effects of metabolic surgery is still unknown.

Some recent studies showed active participation of the intestinal microbiota in the host metabolism and association of the changes in the composition of the gut microbiota with obesity and other metabolic disorders [11]. Moreover, intestinal bacteria are known to play an essential role in the synthesis and metabolism of bile acids, while the latter, in their turn, regulate carbohydrate metabolism [12].

This publication is aimed to summarize current knowledge about the intestinal microbiota in obese people, its role in the host metabolism, along with possible mechanisms of interactions among the gut microflora and metabolic effects of bariatric surgery, with a focus on bile acids as a possible component of such interactions. Despite the fact that some papers, dedicated to specific aspects of the topic, have been published to date $[10,11$, 13-17], we believe that ours is the most comprehensive review covering the diversity of perspectives.

\section{Intestinal microbiota}

Intestinal microbiota is a complex and diverse community of the microorganisms colonizing gastrointestinal tract. The species composition of this community is host-specific. At the same time, it can be altered by various external or internal factors. In humans, the intestinal bacteria are symbionts. They play an important role in various metabolic processes, such as digestion. They can also interfere with energy metabolism pathways by stimulating energy extraction from the diet, and regulate bile acid metabolism [13]. An imbalance in the symbiosis of the gut microbiota (a dysbiosis), for an instance, as a result of a diet rich in fat and low in fibers, may enhance the intestinal permeability and translocation of endotoxin (lipopolysaccharide, LPS) into circulation. The LPS from intestinal bacteria may cause a chronic inflammatory process, leading to obesity, insulin resistance and the metabolic syndrome $[18,19]$.

The healthy human digestive tract microbiota comprises of bacteria, archaea, fungi, viruses and protozoans. Intestinal bacteria include mainly representatives of the seven phyla: Bacteroidetes, Firmicutes, Actinobacteria, Proteobacteria, Verrucomicrobia, Fusobacteria and Spirochaetes. Bacteroidetes and Firmicutes constitute about $90 \%$ of all gastrointestinal bacteria species [14].

Emergence of gene sequencing technology has offered a challenge of new information about gastrointestinal microbiota. Sequencing is a general term of physico-chemical methods for detection of amino acid residues in proteins and nucleotide sequence of nucleic acids. The sequencing of specific regions of $16 \mathrm{~S}$ or $18 \mathrm{~S}$ ribosomal RNA (rRNA) can identify microorganisms and their relative content in the purified DNA. The 16S rRNA was chosen as universal marker for species identification, since it has been found in the genomes of all prokaryotes and was characterized by a very low variability. At present, 16S rRNA gene sequencing is considered the "golden standard" of accurate bacterial species identification. Therefore, the bacterial gene sequencing has become a crucial point in the evaluation of interrelationship between the microbial diversity and different physiological and pathophysiological conditions. It also enabled scientists to observe the differences among the microbial communities in different host phenotypes, diet types and morbidity conditions (e.g., obesity) [20].

\section{Intestinal microbiota vs. obesity}

Some studies have shown that lean vs. overweight/obese patients may demonstrate differences in the composition of their intestinal florae. The overweight or obese people, along with the patients with $\mathrm{DM} 2$, usually have a high ratio of Firmicutes to Bacteroidetes $(\mathrm{F} / \mathrm{B})$, compared with healthy control patients [16, 21]. Some studies report a low diversity of the intestinal bacteria in overweight vs. lean people [22]. On the other hand, some recent publications from the last five years failed to report any body weight depending differences in the microbiota from the upper intestinal tract [16].

Bioinformatics analysis of abundant obtained sequencing helped identifying the so-called alpha $(\alpha-)$ and beta diversity ( $\beta$ diversity) of the intestinal microflora. Alfa-diversity is a species diversity within the same host individual. A high $\alpha$-diversity indicates a wide range of bacterial species in a stool sample. $\beta$ diversity is an inter-individual species diversity, i.e. it characterizes how bacterial species diversity varies among different host individuals. In a recent study, F.G. Wang et al. [21] used 16S ribosomal DNA amplicon sequencing to compare $\alpha$ - and $\beta$ diversities of gut microbiota sampled from 20 healthy volunteers and 26 obese patients with or without DM2. They demonstrated similar $\alpha$-diversity values for both healthy people and obese patients, which matched the results of some other cross-sectional studies $[23,24]$. On the other hand, $\beta$-diversity was higher in morbidly obese patients compared with healthy volunteers. In general, the heterogeneity of the intestinal microbiota in obese patients was higher than in lean control humans. R. Liu et al. in a metagenome-wide association study have also reported a greater diversity of gut microbiota associated with obesity [25].

Many researchers reported lower abundance of commensal intestinal bacteria, such as Faecalibacterium prausnitzii, Bacteroides, Akkermansia muciniphila or Prevotellais, in overweight vs. lean study subjects [15]. The gut microbiota may influence some metabolic processes of the host, including energy balancing, biosynthesis of steroid hormones and bile acid metabolism. Considering the abundance and a wide functional variety of intestinal microbes, it is not surprising that their imbalance promotes a range of pathological conditions. Changes in the composition of intestinal microflora are associated with obesity and $\mathrm{DM} 2$, partly because the microbiota in obese people appears more efficient in terms of harvesting energy from the diet $[10,26]$. The host energy accumulation is mediated by the gut microbiota through the synthesis of short chain fatty acids (SCFA), which are rich sources of energy for the host, and by the influence on the host genome areas in charge of encoding lipoprotein lipase, AMP-activated protein kinase and endocannabioid system [15].

There may be various causes of dysbiosis, including dietary manipulations, stress, genetic predisposition of the host, consumption of antibiotics, infestation with pathogenic bacteria, obesity, DM, some neurological disorders, allergy, and inflammatory bowel diseases [15].

The modification of intestinal microflora can potentially improve such metabolic disorders. This has been illustrated in the study conducted by A. Vrieze et al., in which oral infusion of intestinal microbiota from lean donor mice to those with metabolic syndrome temporarily improved their sensitivity to insulin. The authors also demonstrated the correlation between the gut microbiota and glucose metabolism [27]. 


\section{Gut microbiota vs. bariatric surgery}

Bariatric procedures definitely reduce body weight and improve glucose metabolism, but whether they specifically alter the profile of intestinal microflora is still the matter of discussion. Some recent study with direct comparison of morbidly obese patients before and after RYGB clearly demonstrated the improved diversity and species composition of gut microbiota associated with weight loss and improved glycemia. Moreover, over a half of initially altered bacterial species kept their relative abundances for a year after the surgery [28]. The intestinal flora in post-RYGB patients became significantly restructured in six months following the procedure compared with lean subjects or obese patients [22]. Hence, bariatric surgery can definitely cause beneficial and persistent changes in intestinal microbial community. Body mass reduction due to life-style modifications and calorie-restricted diets is also associated with certain changes in gut microbiota [29]. However, it is bariatric surgery, rather than the low-calorie diet, which shifts the profile of intestinal microflora towards the profile observed in lean people [30].

Specifically, patients after the gastric bypass compared with their pre-surgical condition, or with normal-weight control subjects, had significantly increased numbers of Proteobacteria and Bacteroidetes and decreased amounts of Firmicutes [22, 28, 30]. The Bacteroidetes to Firmicutes ratio may also increase after he bariatric surgery [30]. Contradicting results were reported by some other studies, in which Bacteroidetes dropped down in numbers after RYGB, while Firmicutes increased their quantities $[31,32]$. This controversy can be attributed to the differences in study protocols (e.g., sample sizes) and in the degrees of caloric intake reduction. J.P. Furet et al. have shown that the higher proportion of Bacteroides in post-RYGB patients was associated with greater reductions in both body weights and post-surgery leptin levels [33]. In contrast to RYGB, a laparoscopic sleeve gastrectomy, conducted on both animals and humans, yielded an increase of Bacteroidetes numbers with reduced quantities of Firmicutes, as well as in restoration of the gut microbiota diversity $[31,34,35]$.

As for changes in other taxonomic bacterial units after bariatric procedures, the results of the studies on human subjects and rodents are also quite controversial. In their systematic review, Yan Guo et al. analyzed the data of 21 studies (twelve on animals and nine on human subjects) including 11 top quality research projects (six on animals and five on humans) [16]. Those strongly demonstrated that the phylum Proteobacteria and its class Gammaproteobacteria, order Enterobacteriales, family Enterobacteriaceae, and genus Escherichia increased numerically after the bariatric procedures. The increased abundances of some genera (Citrobacter, Enterobacter, Escherichia and Salmonella) and species (Emeyrobacter cancerogenus, E. hormaechei, Escherichia coli, Klebsiella pneumoniae, and Salmonella enterica) from the Enterobacteriaceae family were detected with a strong or moderate evidence. Among the order Clostridiales (the dominant order among Firmicutes), seven genera (Anaerostipes, Clostridium, Faecalibacterium, Blautia, Coprococcus, Dorea, and Ruminococcus) and two families (Clostridiaceae and Ruminococcaceae) declined numerically after the surgery with a strong or moderate evidence as well [16].

Fu-Gang Wang et al. demonstrated an increased $\alpha$-diversity following SG and RYGB, even though in RYGB group these changes did not reach the level of statistical significance. The increased post-surgery $\alpha$-diversity is potentially related to reduced gastric acid secretion and increased dissolved oxygen concentration in distal intestine [21]. As for $\beta$-diversity, a few studies reported its alterations after bariatric procedures. D.A. Medina et al. [36] and Fu-Gang Wang et al. [21] observed some reduction of $\beta$-diversity following the SG, while Guo Y et al. did not [37].

Changes in the species composition of intestinal bacteria after SG or RYGB may occur as a response to reduced gastric volume and anatomical reorganization of intestinal tract. For instance, reduced gastric acid secretion yields $\mathrm{pH}$ increase in the lower parts of the intestinal tract, particularly in the colon. Therefore, the chyme, altered by incomplete digestion, may change the intestinal medium and diversity of intestinal flora.

Two types of bariatric surgery cause different gut microbiota changes. For example, R. Murphy et al. discovered that RYGB produced a decrease of Bacteroidetes and increase of both Firmicutes and Actinobacteria in their study of intestinal microflora following the laparoscopic RYGB or SG in obese patients with DM2 [31]. In contrast, SG surgery caused an increase in Bacteroidetes numbers. At a species level, solely an increase in Roseburia intestinalis from the phylum Firmicutes was the only compositional change common to both types of bariatric surgery among the patients with diabetes remission. These results were rather surprising, because they differed distinctly from any other previously published, and because SG was found by another study, conducted on rodents, to yield smaller changes in gut microbiota than RYGB, possibly, due to less aggressive anatomical alterations associated with SG [37].

Summarizing the above findings, we should mention that bariatric surgery, particularly RYGB, may cause rapid and persistent changes in obese people. The question is whether such changes are related to the postoperative metabolic improvements. In an animal study by A.P. Liou et al., the transfer of the gut microbiota from RYGB-treated mice to unoperated, germ-free mice resulted in weight loss and decreased fat mass in the recipient animals, relative to the microbiota recipients after the sham surgery [38]. Moreover, fecal transplantation from postRYGB obesity patients to germ-free mice also resulted in fat mass loss in their bodies [32], which could be indicative of the fact that the alteration of microbial profile in distal intestine was a causative factor of the weight loss after RYGB.

\section{Importance of bile acids in bariatric surgery}

Altered metabolism of bile acids and their relationship with gut microbiota is an important component of bariatric surgery effects. There is some evidence that bariatric procedures result in increased level of circulating bile acids. Moreover, bile acid concentrations negatively correlate with postprandial glucose level and positively correlate with peak secretion of glucagon-like peptide 1 (GLP-1), implying the possible role of bile acids in improving glucose metabolism after the obesity surgery [10, 39, 40].

Possible mechanisms of increased bile acid levels in blood serum after bariatric surgery

Both types of bariatric surgery cause either anatomical or functional changes in upper gastrointestinal tract, possibly affecting the enterohepatic circulation of bile acids and elevating their levels in blood serum. 
Bile acids are synthesized from cholesterol in the liver, stored in the gallbladder, and excreted into the duodenum in response to enteral stimuli. About $95 \%$ of luminal bile acids undergo enterohepatic circulation: they come back into the hepatic portal vein from the terminal ileum and are reutilized by the liver. RYGB shortens the enterohepatic circulation and thus promotes the contact of luminal bile acids with the ileum, resulting in their early and rapid reabsorption. The length ratio of the biliopancreatic limb to the common limb is presumed to explain elevated postoperative bile acid concentrations in blood serum [17].

Only limited data are available, regarding post-SG levels of serum bile acids. While one study on human subjects has demonstrated an immediate increase in serum concentrations of bile acids after the surgery [41], two other studies were able to demonstrate solely their statistically nonsignificant postoperative elevation after one or two years [42, 43]. In contrast, studies conducted on animals, have shown consistently increased serum concentrations of bile acids following SG $[40,44]$. Due to minimal direct anatomical manipulation on the intestine, the elevated bile acid concentrations in serum after SG are likely to be a secondary consequence of altered gastrointestinal function and progressive intestinal adaptation. SG promotes gastric motility and bowel movement. It is also associated with reduced bile acid synthesis in the liver, enlarged intestinal villi length, and augmented bile acid reabsorption intestinal area, which might explain the increased serum concentrations of bile acids after SG [44]

Mechanisms of relationship between bile acids and metabolic effects of bariatric surgery

Enterohepatic regulation of bile acids metabolism is mediated via FXR-FGF19-CYP7A1 system. Through the nuclear farnesoid $X$ receptor (FXR, NR1H4), bile acids activate the secretion of fibroblast growth factor 19 (FGF19, or FGF15 in rodents) in human ileum, which, in its turn, interacts with hepatic fibroblast growth factor receptor-4 (FGFR4) within the portal venous system and inhibits the CYP7A1 expression (a rate-limiting enzyme for bile acid synthesis in the liver).

Some experimental studies confirmed the beneficial effects of FXR on lipid metabolism, carbohydrate metabolism and insulin sensitivity [17]. Since bile acids are natural ligands for FXR, it is believed that the "bile acids - FXR" pathway may contribute to the metabolic effects of bariatric surgery. There is still no consensus on the issue, because the available data on FXR, FGF19, and their associations with postoperative metabolic parameters give controversial results [17].

G protein-coupled receptor 1, also known as TGR5 (GPR131), is a membrane-type receptor for bile acids. The activation of TGR5 in enteroendocrine $L$ cells of the distal small intestine in response to the action of intraluminal bile acids is considered the most important signaling pathway for stimulating GLP-1 secretion in both animals and humans. Postoperative GLP-1 levels increase in blood serum simultaneously with increased concentrations of luminal bile acids could be an important (although one of many) mechanism of improving glucose tolerance following the bariatric surgery [45].

In skeletal muscles and brown adipose tissue, the elevated levels of bile acids in serum promote thyroxine (T4) conversion into triiodothyronine (T3) viaTGR5. This process stimulates energy expenditure, and also may contribute to the weight loss [46, 47]. Yet, the energy balance is a very complex process, connected with multiple factors, such as body weight, physical exercises, hormones, etc. That is why the role of TGR5-mediated energy regulation after bariatric surgeries definitely needs further investigation.

In the study conducted on the patients with morbid obesity, the circulating bile acid concentrations decreased one week after the bariatric surgery, then went up slightly in three months, and then showed a drastic surge in 12 months. However, the augmented peptide YY and GLP-1 levels in serum, along with improved metabolic control, were seen after a week and sustained within 12 months after the surgery [48]. Therefore, stepwise elevation of bile acid concentrations in blood serum do not precisely match rapid metabolic improvements, observed soon after the weight loss surgery, hence further studies are needed to elucidate their signaling pathways.

\section{Microbiota vs. bile acids}

Bile acids and gut microbiota are mutually altered by bariatric surgery. Gut microflora plays a key role in a synthesis and biotransformation of bile acids. The biosynthesis of bile acids comprises of several enzymatic catalytic reactions. S.I. Sayin et al. demonstrated lowered hepatic expression of many enzymes, including 7 alpha-hydroxylase and sterol 27-hydroxylase, in conventionally raised mice, compared with germ-free control animals, proving the role of the gut microbiota in regulation of bile acids synthesis [49]. After secretion into the intestine, primary bile acids are further metabolized by the intestinal microflora into secondary bile acids (i.e., cholic acid into deoxycholic acid, chenodeoxycholic acid into lithocholic acid, etc.), resulting in chemical diversity of the bile. The process of microbial deconjugation is catalyzed by bile salt hydrolase (BSH) enzyme. With a metagenome analysis, I.M. Jones et al. confirmed a wide expression of BSH in human gut microbiota [50]. Primary deconjugated bile acids are also transformed into secondary bile acids via microbial $7 \alpha / \beta$-dehydroxylation pathway. The $7 \alpha / \beta$ dehydroxylation activity was found in some intestinal bacteria, such as Clostridium spp. [51].

In their turn, the intestinal concentration and composition of bile affect the gut microbiota. Increased luminal concentration of bile acids produce an antibacterial effect, killing some bacterial strains and prompting the growth of others (e.g., Bilophila wadsworthia). Additionally, some particular bile acids are able to activate the intestinal FXR, causing an enteroprotective effect due to inhibition of bacterial growth in the ileum [17]. All these data validate close interactions between the gut microflora and intestinal bile acids.

The relative abundance of some bacteria, such as Bacteroides and Roseburia, was significantly altered in post-SG wild-type mice, compared with sham-operated animals, but did not changed after the surgery in FXR knockout mice, which did not demonstrate any post-SG weight and metabolic improvements, including glucose tolerance [12]. Therefore, metabolic effect of SG may be mediated by bile acids and by transmission of FXR and gut microbiota signals.

These data indicate that obesity-targeting surgery may affect the interactions between bile acids and the intestinal microflora, thus providing postoperative metabolic benefits. 


\section{Conclusion}

Drastic metabolic effects of bariatric surgery increase the possibility of DM2 remission in obese patients. The surgery improves glucose metabolism and offers the challenge in understanding of pathophysiology of metabolic disorders rather than reducing the body weight alone. Available studies have demonstrated that both bile acid levels and the composition of intestinal microbiota change after the bariatric procedures and may be associated with the postoperative metabolicc improvements. The precise mechanisms of these interactions, causing beneficial effects of bariatric surgery, should be clarified in follow-up studies.

\section{Conflicts of Interest}

The authors declare no conflicts of interest.

\section{References}

1. Ng M, Fleming $T$, Robinson $M$, Thomson B, Graetz N, Margono C, et al. Global, regional, and national prevalence of overweight and obesity in children and adults during 1980-2013: a systematic analysis for the Global Burden of Disease Study 2013. Lancet 2014; 384(9945): 766781. https://doi.org/10.1016/s0140-6736(14)60460-8.

2. World Health Organization. Fact sheet. Obesity and overweight. 2020. https://www.who.int/en/news-room/fact-sheets/detail/obesity-andoverweight.

3. Field AE, Coakley EH, Must A, Spadano JL, Laird N, Dietz WH, et al. Impact of overweight on the risk of developing common chronic diseases during a 10-year period. Arch Intern Med. 2001; 161(13): 1581-1586. https://doi.org/10.1001/archinte.161.13.1581.

4. International Diabetes Federation. IDF diabetes Atlas, 7th ed Brussels: International Diabetes Federation, 2015: 140p. https://diabetesatlas.org/upload/resources/previous/files/7/IDF\%20Di abetes\%20Atlas\%207th.pdf.

5. Mason EE. History of obesity surgery. Surg Obes Relat Dis. 2005; 1(2): 123-125. https://doi.org/10.1016/j.soard.2005.01.005.

6. Pories WJ, Caro JF, Flickinger EG, Meelheim HD, Swanson MS. The control of diabetes mellitus (NIDDM) in the morbidly obese with the Greenville Gastric Bypass. Ann Surg 1987; 206(3): 316-323. https://doi.org/10.1097/00000658-198709000-00009.

7. Mingrone G, Panunzi S, De Gaetano A, Guidone C, laconelli A, Nanni G, et al. Bariatric-metabolic surgery versus conventional medical treatment in obese patients with type 2 diabetes: 5 year follow-up of an open-label, single-centre, randomised controlled trial. Lancet 2015; 386(9997): 964-973. https://doi.org/10.1016/s0140-6736(15)00075-6.

8. Mehaffey JH, LaPar DJ, Clement KC, Turrentine FE, Miller MS, Hallowell PT, et al. 10-Year Outcomes After Roux-en-Y Gastric Bypass. Ann Surg 2016; 264(1): 121-126. https://doi.org/10.1097/sla.0000000000001544.

9. Schauer PR, Bhatt DL, Kirwan JP, Wolski K, Brethauer SA, Navaneethan $\mathrm{SD}$, et al. Bariatric surgery versus intensive medical therapy for diabetes-3-year outcomes. N Engl J Med 2014; 370(21): 2002-2013. https://doi.org/10.1056/nejmoa1401329.

10. Liu H, Hu C, Zhang X, Jia W. Role of gut microbiota, bile acids and their cross-talk in the effects of bariatric surgery on obesity and type 2 diabetes. J Diabetes Investig 2018; 9(1): 13-20. https://doi.org/10.1111/idi.12687.

11. Hartstra AV, Bouter KE, Bäckhed F, Nieuwdorp M. Insights into the role of the microbiome in obesity and type 2 diabetes. Diabetes Care 2015; 38(1): 159-165. https://doi.org/10.2337/dc14-0769.

12. Ryan KK, Tremaroli V, Clemmensen C, Kovatcheva-Datchary $P$, Myronovych A, Karns R, et al. FXR is a molecular target for the effects of vertical sleeve gastrectomy. Nature 2014; 509 (7499): 183-188. https://doi.org/10.1038/nature13135.
13. Cani PD, Osto M, Geurts L, Everard A. Involvement of gut microbiota in the development of low-grade inflammation and type 2 diabetes associated with obesity. Gut Microbes 2012; 3(4):279-288. https://doi.org/10.4161/gmic.19625.

14. Castaner O, Goday A, Park YM, Lee SH, Magkos F, Shiow STE, et al. The Gut Microbiome Profile in Obesity: A Systematic Review. Int J Endocrinol 2018; 2018: 4095789 https://doi.org/10.1155/2018/4095789.

15. Tabasi M, Ashrafian F, Khezerloo JK, Eshghjoo S, Behrouzi A, Javadinia SA, et al. Changes in Gut Microbiota and Hormones After Bariatric Surgery: a Bench-to-Bedside Review. Obes Surg 2019; 29(5): $1663-$ 1674. https://doi.org/10.1007/s11695-019-03779-7.

16. Guo Y, Huang ZP, Liu CQ, Qi L, Sheng Y, Zou DJ. Modulation of the gut microbiome: a systematic review of the effect of bariatric surgery. Eur J Endocrinol 2018; 178(1): 43-56. https://doi.org/10.1530/eje-17-0403.

17. Wang W, Cheng Z, Wang Y, Dai Y, Zhang X, Hu S. Role of Bile Acids in Bariatric Surgery. Front Physiol 2019; 10: 374. https://doi.org/10.3389/fphys.2019.00374.

18. Tsukumo DM, Carvalho BM, Carvalho Filho MA, Saad MJ. Translational research into gut microbiota: newhorizons on obesity treatment: updated 2014. Arch Endocrinol Metab 2015; 59(2): 154-160. https://doi.org/10.1590/2359-3997000000029.

19. Moran CP, Shanahan F. Gut microbiota and obesity: role in aetiology and potential therapeutic target. Best Pract Res Clin Gastroenterol 2014; 28(4): 585-597. https://doi.org/10.1016/j.bpg.2014.07.005

20. Duncan SH, Lobley GE, Holtrop G, Ince J, Johnstone AM, Louis P, et al. Human colonic microbiota associated with diet, obesity and weight loss. Int J Obes (Lond) 2008; 32(11): 1720-1724. https://doi.org/10.1038/ijo.2008.155.

21. Wang FG, Bai RX, Yan WM, Yan M, Dong LY, Song MM. Differential composition of gut microbiota among healthy volunteers, morbidly obese patients and post-bariatric surgery patients. Exp Ther Med 2019; 17(3): 2268-2278. https://doi.org/10.3892/etm.2019.7200.

22. Zhang $H$, DiBaise JK, Zuccolo A, Kudrna D, Braidotti $M, Y u Y$, et al. Human gut microbiota in obesity and after gastric bypass. Proc Natl Acad Sci U S A 2009; 106(7): 2365-2370. https://doi.org/10.1073/pnas.0812600106.

23. López-Contreras $B E$, Morán-Ramos $S$, Villarruel-Vázquez $R$, MacíasKauffer L, Villamil-Ramírez $\mathrm{H}$, León-Mimila $\mathrm{P}$, et al. Composition of gut microbiota in obese and normal-weight Mexican school-age children and its association with metabolic traits. Pediatr Obes 2018; 13(6) 381-388. https://doi.org/10.1111/ijpo.12262.

24. Hakkak R, Korourian S, Foley SL, Erickson BD. Assessment of gut microbiota populations in lean and obese Zucker rats. PLoS One 2017; 12(7): e0181451. https://doi.org/10.1371/journal.pone.0181451.

25. Liu R, Hong J, Xu X, Feng $Q$, Zhang $D, G u$ Y, et al. Gut microbiome and serum metabolome alterations in obesity and after weight-loss intervention. Nat Med 2017; 23(7): 859-868. https://doi.org/10.1038/nm.4358.

26. Turnbaugh PJ, Ley RE, Mahowald MA, Magrini V, Mardis ER, Gordon JI. An obesity-associated gut microbiome with increased capacity for energy harvest. Nature 2006; 444(7122): 1027-1031. https://doi.org/10.1038/nature05414.

27. Vrieze A, Van Nood E, Holleman F, Salojärvi J, Kootte RS, Bartelsman $J F$, et al. Transfer of intestinal microbiota from lean donors increases insulin sensitivity in individuals with metabolic syndrome. Gastroenterology 2012; 143(4): 913-916. https://doi.org/10.1053/j.gastro.2012.06.031.

28. Palleja A, Kashani A, Allin KH, Nielsen T, Zhang C, Li Y, et al. Roux-en-Y gastric bypass surgery of morbidly obese patients induces swift and persistent changes of the individual gut microbiota. Genome Med 2016; 8(1): 67. https://doi.org/10.1186/s13073-016-0312-1.

29. Nadal I, Santacruz A, Marcos A, Warnberg J, Garagorri JM, Moreno LA, et al. Shifts in clostridia, bacteroides and immunoglobulin-coating fecal 
bacteria associated with weight loss in obese adolescents. Int J Obes (Lond) 2009; 33(7):758-767. https://doi.org/10.1038/ijo.2008.260.

30. Damms-Machado A, Mitra S, Schollenberger AE, Kramer KM, Meile T, Königsrainer $A$, et al. Effects of surgical and dietary weight loss therapy for obesity on gut microbiota composition and nutrient absorption. Biomed Res Int 2015; 2015: 806248. https://doi.org/10.1155/2015/806248.

31. Murphy R, Tsai $P$, Jüllig $M$, Liu A, Plank L, Booth $M$. Differential changes in gut microbiota after gastric bypass and sleeve gastrectomy bariatric surgery vary according to diabetes remission. Obes Surg 2017; 27(4): 917-925. https://doi.org/10.1007/s11695-016-2399-2.

32. Tremaroli V, Karlsson F, Werling $M$, Ståhlman M, Kovatcheva-Datchary $P$, Olbers $T$, et al. Roux-en- $Y$ gastric by-pass and vertical banded gastroplasty induce long-term changes on the human gut microbiome contributing to fat mass regulation. Cell Metab 2015; 22(2): 228-238. https://doi.org/10.1016/j.cmet.2015.07.009.

33. Furet JP, KongLC, Tap J, Poitou C, Basdevant A, Bouillot JL, et al. Differential adaptation of human gut microbiota to bariatric surgeryinducedweight loss: links with met-abolic and low-grade inflammation markers. Diabetes 2010; 59(12): 3049-3057. https://doi.org/10.2337/db10-0253.

34. Jahansouz C, Staley C, Bernlohr DA, Sadowsky MJ, Khoruts A, Ikramuddin $\mathrm{S}$, et al. Sleeve gastrectomy drives persistent shifts in the gut microbiome. Surg Obes Relat Dis 2017; 13(6): 916-924. https://doi.org/10.1016/j.soard.2017.01.003.

35. Shao Y, Shen Q, Hua R, Evers SS, He K, Yao Q, et al. Effects of sleeve gastrectomy on the composition and diurnal oscillation of gut microbiota related to the metabolic improvements. Surg Obes Relat Dis 2018; 14(6): 731-739. https://doi.org/10.1016/j.soard.2018.02.024.

36. Medina DA, Pedreros JP, Turiel D, Quezada N, Pimentel F, Escalona A, et al. Distinct patterns in the gut microbiota after surgical or medical therapy in obese patients. PeerJ 2017; 5: e3443. https://doi.org/10.7717/peerj.3443.

37. Guo Y, Liu CQ, Shan CX, Chen Y, Li HH, Huang ZP, et al. Gut microbiota after Roux-en- $Y$ gastric bypass and sleeve gastrectomy in a diabetic rat model: Increased diversity and associations of discriminant genera with metabolic changes. Diabetes Metab Res Rev 2017; 33(3). https://doi.org/10.1002/dmrr.2857.

38. Liou AP, Paziuk M, Luevano JM Jr, Machineni S, Turnbaugh PJ, Kaplan LM. Conserved shifts in the gut microbiota due to gastric bypass reduce host weight and adiposity. Sci Transl Med 2013; 5(178): 178ra41. https://doi.org/10.1126/scitranslmed.3005687.

39. Jahansouz C, Xu H, Hertzel AV, Serrot FJ, Kvalheim N, Cole A, et al. Bile Acids Increase Independently From Hypocaloric Restriction After Bariatric Surgery. Ann Surg 2016; 264: 1022-1028. https://doi.org/10.1097/sla.0000000000001552.

40. Myronovych A, Kirby M, Ryan KK, Zhang W, Jha P, Setchell KD, et al. Vertical sleeve gastrectomy reduces hepatic steatosis while increasing serum bile acids in a weight-loss-independent manner. Obesity (Silver Spring) 2014; 22(2): 390-400. https://doi.org/10.1002/oby.20548.

41. Kindel TL, Krause C, Helm MC, McBride CL, Oleynikov D, Thakare R, et al. Increased glycine-amidated hyocholic acid correlates to improved early weight loss after sleeve gastrectomy. Surg Endosc 2018; 32(2): 805-812. https://doi.org/10.1007/s00464-017-5747-y.

42. Nakatani $H$, Kasama K, Oshiro $T$, Watanabe $M$, Hirose $H$, Itoh $H$. Serum bile acid along with plasma incretins and serum high-molecular weight adiponectin levels are increased after bariatric surgery. Metabolism 2009; 58(10): 1400-1407. https://doi.org/10.1016/j.metabol.2009.05.006

43. Haluzíková D, Lacinová Z, Kaválková P, Drápalová J, Křížová J, Bártlová $M$, et al. Laparoscopic sleeve gastrectomy differentially affects serum concentrations of FGF-19 and FGF-21 in morbidly obese subjects. Obesity (Silver Spring) 2013; 21(7): 1335-1342. https://doi.org/10.1002/oby.20208.
44. Cummings BP, Bettaieb A, Graham JL, Stanhope KL, Kowala M, Haj FG, et al. Vertical sleeve gastrectomy improves glucose and lipid metabolism and delays diabetes onset in UCD-T2DM rats. Endocrinology 2012; $\quad$ 153(8): $3620-3632$ https://doi.org/10.1210/en.2012-1131.

45. Madsbad S, Holst JJ. GLP-1 as a mediator in the remission of type 2 diabetes after gastric bypass and sleeve gastrectomy surgery. Diabetes 2014; 63(10): 3172-3174. https://doi.org/10.2337/db14-0935.

46. Kohli R, Bradley D, Setchell KD, Eagon JC, Abumrad N, Klein S. Weight loss induced by Roux-en- $Y$ gastric bypass but not laparoscopic adjustable gastric banding increases circulating bile acids. J Clin Endocrinol Metab 2013; 98(4): E708-E712. https://doi.org/10.1210/jc.2012-3736.

47. Broeders EP, Nascimento EB, Havekes B, Brans B, Roumans KH, Tailleux $A$, et al. The bile acid chenodeoxycholic acid increases human brown adipose tissue activity. Cell Metab 2015; 22(3): 418-426. https://doi.org/10.1016/i.cmet.2015.07.002.

48. Steinert RE, Peterli R, Keller S, Meyer-Gerspach AC, Drewe J, Peters T, et al. Bile acids and gut peptide secretion after bariatric surgery: a 1year prospective randomized pilot trial. Obesity (Silver Spring) 2013; 21(12): E660-E668. https://doi.org/10.1002/oby.20522.

49. Sayin SI, Wahlström A, Felin J, Jäntti S, Marschall HU, Bamberg K, et al. Gut microbiota regulates bile acid metabolism by reducing the levels of tauro-beta-muricholic acid, a naturally occurring FXR antagonist. Cell Metab 2013; 17(2): 225-235. https://doi.org/10.1016/j.cmet.2013.01.003.

50. Jones BV, Begley M, Hill C, Gahan CG, Marchesi JR, et al. Functional and comparative metagenomic analysis of bile salt hydrolase activity in the human gut microbiome. Proc Natl Acad Sci U S A 2008; 105(36): 13580-13585. https://doi.org/10.1073/pnas.0804437105.

51. Dawson PA, Karpen SJ. Intestinal transport and metabolism of bile acids. J Lipid Res 2015; 56(6): 1085-1099. https://doi.org/10.1194/jlr.r054114.

\section{Authors:}

Marina A. Berkovskaya - MD, PhD, Instructor, Department of Endocrinology No.1, N.V. Sklifosovsky Institute of Clinical Medicine, I.M. Sechenov First Moscow State Medical University (Sechenov University), Moscow, Russia. https://orcid.org/0000-0003-4974-7765.

Yulia P. Sych - MD, PhD, Instructor, Department of Endocrinology No.1, N.V. Sklifosovsky Institute of Clinical Medicine, I.M. Sechenov First Moscow State Medical University (Sechenov University), Moscow, Russia. https://orcid.org/0000-0002-7000-0095.

Olesya Yu. Gurova - MD, PhD, Assistant Professor, Department of Endocrinology No.1, N.V. Sklifosovsky Institute of Clinical Medicine, I.M. Sechenov First Moscow State Medical University (Sechenov University), Moscow, Russia. https://orcid.org/0000-0003-2576-4421.

Valentin V. Fadeev - MD, DSc, Professor, Corresponding Member of RAS, Chair of the Department of Endocrinology No.1, N.V. Sklifosovsky Institute of Clinical Medicine, I.M. Sechenov First Moscow State Medical University (Sechenov University). https://orcid.org/0000-0002-3026-6315. 